\title{
Developments in chemotherapy for the treatment of acute myeloid leukemia
}

\author{
Mark W Drummond*,1 \\ ${ }^{1}$ Beatson Cancer Centre, Glasgow, UK, G12 OYN \\ *Author for correspondence: mark.drummond@ggc.scot.nhs.uk
}

Mark Drummond obtained his medical degree from the University of Glasgow (UK) and since then has trained as a hematologist in the West of Scotland and the Canterbury District Health Board (Christchurch, New Zealand) and back at Glasgow University, where he also gained a PhD in chronic myeloid leukemia. He is currently a consultant hematologist and honorary senior lecturer at the Beatson Cancer Centre (Glasgow, UK) as well as an investigator on multiple UK and international clinical trials.

Here he talks to Commissioning Editor Jennifer Straiton, commenting on the recent announcement from the Scottish Medicines Consortium, regarding their acceptance of the use of the combination chemotherapy treatment $V_{y x e o s}{ }^{\circledR}$ (daunorubicin and cytarabine) for the treatment of high-risk acute myeloid leukemia in adults. He then discusses the ongoing and future clinical trials in this disease area and then turns to more personalized treatments.

First draft submitted: 24 April 2019; Accepted for publication: 24 April 2019; Published online: 11 July 2019

Keywords: chemotherapy $\bullet$ combination therapies $\bullet$ myeloid disease $\bullet$ treatment options

\section{Could you introduce yourself \& give a bit of detail about the SMC's recent announcement?}

I am a consultant hematologist at the Beatson West of Scotland Cancer Centre (Glasgow, UK) with an interest in myeloid disorders, particularly acute myeloid leukemia (AML), myelodysplastic syndrome (MDS) and myeloproliferative disorders (MPN). The SMC decision is very welcome; I think that it introduces a treatment option for this specific group of patients who, until now, have had little available to them. The approval given for the licensed indication, with no other restrictions, is a very welcome decision.

\section{What clinical impact do you think the acceptance of Vyxeos ${ }^{\circledR}$ will have?}

I think there will be a number of positives, the first being that it will force us to adopt a more patient-specific treatment approach at first diagnosis of AML. Recently there have been multiple new drugs approved for clinical use and the notion that one size of treatment fits all AML patients is no longer the case. This is something that we have known for a while, but we just have not had the treatment options to work around it. Now that we do it means that stratifying and classifying these patients at diagnosis is more important than ever before and can allow us to tailor the treatment to the individual.

There are benefits to be had from the patient's perspective, potentially improving both outcome and experience. The trial has shown that more patients will get to transplant, leading to a far better outcome and overall survival rates, and we are seeing that this drug leads to higher rates of complete remission, something we know to be associated with beneficial effects on quality of life. The hope is that this would translate into a better patient experience and well-being, factors which are likely to play a role in further improving outcome. The toxicity of the drug is a bit different from what we are used to with the conventional approach; we are seeing less alopecia and more cytopenias, something which may reflect the more targeted efficacy of the drug.

I would hope that, at least for the post induction cycles, we can think about potentially delivering this treatment as an outpatient as well, which is something that we have not been able to do before. This is something that could also feed into a better patient experience and is better for the health service, it would involve less resource use, less in-patient bed usage and so on. 


\section{Do you see Vyxeos being approved for other forms of cancer in the near future?}

The formulation that has been identified here has been developed primarily on models of AML; this very specific molar ratio of the two chemotherapy drugs has been designed to optimize drug delivery and drug concentration within the AML cells. However, following the success shown in treating AML, it is being trialed in other tumor types, particularly those currently treated by anthracyclines; there are trials currently going on in Hodgkin lymphoma and in diffuse large B-cell lymphoma, with potential for use in treating acute lymphoblastic leukemia. The main focus will be on its use in the relapse/refractory settings, with additional trials going on with AML patients in this stage as well. It will be important to look for efficacy signals and safety data in the various tumor types.

There could also be potential for its use in patients with MDS; in about a quarter of cases MDS will transform into AML; therefore, it is logical to see whether Vyxeos will have a role in that group of patients. These are a difficult group of patients who often rely on stem cell transplantation to maintain remission so Vyxeos may have a significant role in their treatment.

\section{How would you say Vyxeos \& the more traditional chemotherapy treatments compare to the newer immunotherapy or CAR-T-based approaches?}

Right now, we do not have the CAR-T option in myeloid disease, CAR-T is really within the lymphoma setting and in lymphoid disorders, specifically acute lymphoblastic leukemia and diffuse large B-cell lymphoma. While there are data coming through on CAR-T use in myeloid disorders, it is quite difficult to get an antigen to target the cancerous cells in myeloid disorders that does not also wipe out the marrow. Patients who have been treated using CAR-T then require allogeneic stem cell transplants to account for the consequences, meaning it is not a viable treatment in a routine sense. Despite multiple tests experimentally, immunotherapies have not really found their way into the clinical myeloid setting.

\section{What further advances do you see with respect to novel chemotherapies in the coming years?} In the case of AML, the treatment landscape has changed remarkably in just a couple of years and is set to change even more in the next couple. I think the big thing coming through in AML which could have a bearing on Vyxeos, particularly when we are looking at combination therapies, is the use of venetoclax, a BCL-2 inhibitor which is currently licensed in chronic lymphocytic leukemia; this is producing some very exciting results in the nonintensive AML-treated population. There is a trial going on now looking at the use of venetoclax in combination with Vyxeos, these are agents that are very deliverable to patients as stand-alone drugs and we would hope are more effective when in combination arms.

I think that the big step in terms of intensively treated patients in AML will be to combine intensive chemotherapy with newer agents, with an ultimate goal of identifying chemotherapy-free treatment pathways. The combination of Vyxeos with more targeted treatments such as FLT-3 inhibitors or isocitrate dehydrogenase inhibitors will likely be an interesting development. FLT-3 inhibitors have only recently entered routine use with the licensing of midostaurin in first-line AML; however, there are three or four new drugs coming along and this next generation of FLT-3 inhibitors could be of great interest. FLT-3-mutated patients are a particularly difficult subgroup of AML patients to treat and have a high relapse rate; there is a suggestion that this combination therapy of Vyxeos and FLT-3 inhibitors could be particularly active in these patients, something that could be very valuable.

Up until fairly recently, the AML community has been predominantly trialing different combinations of conventional chemotherapy and I think Vyxeos is the perfect example of the output of that approach. The two drugs contained in the Vyxeos molecule are ones that we have been using for over 40 years in AML, yet this is probably the first breakthrough in terms of the dose schedule and formulation we have had so far. Lots of variations on the theme have been tried over the years and we are still using a chemotherapy back-bone for the intensively treated patients, a treatment that looks to stay for the foreseeable future. The direction we are going in now is to combine this backbone with some of the newer agents, such as the IDH drugs or the FLT 3-targeted drugs, optimizing chemotherapy and optimizing use of targeted therapies where relevant.

\section{Disclaimer}

The opinions expressed in this interview are those of Mark Drummond and do not necessarily reflect the views of Future Medicine Ltd. 
Financial \& competing interests disclosure

The author has no relevant affiliations or financial involvement with any organization or entity with a financial interest in or financial conflict with the subject matter or materials discussed in the manuscript. This includes employment, consultancies, honoraria, stock ownership or options, expert testimony, grants or patents received or pending, or royalties.

No writing assistance was utilized in the production of this manuscript.

\section{Open access}

This work is licensed under the Attribution-NonCommercial-NoDerivatives 4.0 Unported License. To view a copy of this license, visit http://creativecommons.org/licenses/by-nc-nd/4.0/ 